\title{
Bilinear R-parity violation with flavor symmetry
}

\author{
F. Bazzocchi, ${ }^{a}$ S. Morisi, ${ }^{b}$ E. Peinado, ${ }^{b}$ J.W.F. Valle ${ }^{b}$ and A. Vicente $^{c}$ \\ ${ }^{a}$ International School for Advanced Studies (SISSA), \\ via Bonomea 265, Trieste, Italy \\ ${ }^{b}$ AHEP Group, Instituto de Física Corpuscular - C.S.I.C./Universitat de València, \\ Edificio de Institutos de Paterna, Apartado 22085, E-46071 València, Spain \\ ${ }^{c}$ Laboratoire de Physique Théorique, CNRS - UMR 8627, Université de Paris-Sud 11, \\ F-91405 Orsay Cedex, France \\ E-mail: fbazzo@sissa.it, morisi@ific.uv.es, epeinado@ific.uv.es, \\ valle@ific.uv.es, avelino.vicente@th.u-psud.fr
}

ABSTRACT: Bilinear R-parity violation (BRPV) provides the simplest intrinsically supersymmetric neutrino mass generation scheme. While neutrino mixing parameters can be probed in high energy accelerators, they are unfortunately not predicted by the theory. Here we propose a model based on the discrete flavor symmetry $A_{4}$ with a single R-parity violating parameter, leading to (i) correct Cabbibo mixing given by the Gatto-Sartori-Tonin formula, and a successful unification-like b-tau mass relation, and (ii) a correlation between the lepton mixing angles $\theta_{13}$ and $\theta_{23}$ in agreement with recent neutrino oscillation data, as well as a (nearly) massless neutrino, leading to absence of neutrinoless double beta decay.

Keywords: Beyond Standard Model, Neutrino Physics, Supersymmetric Standard Model, Discrete and Finite Symmetries 


\section{Contents}

1 Introduction 1

2 Bilinear R-parity violation 2

3 The flavored BRpV model 4

4 Large $\theta_{13}$ and deviations from maximal atmospheric mixing $\quad 7$

$\begin{array}{lll}5 & \text { Scalar potential and spectrum } & 7\end{array}$

6 Discussion and conclusions 12

\section{Introduction}

Significant progress has recently been made at the Large Hadron Collider (LHC), with the discovery of a new scalar state in the $125 \mathrm{GeV}$ mass region [1,2]. Although a conclusive identification is still not possible, the properties of this new state resemble very much those expected for the long-awaited Higgs boson. This already constitutes one of the most important discoveries of modern physics and represents an incredible success for a 50-year old theory.

If this new scalar state were confirmed to be the Higgs boson, we would know that the Standard Model (SM) is indeed the correct effective description of elementary particles at least up to a scale which we still ignore. Measuring the exact Higgs boson mass would be crucial to know up to which scale the Higgs boson scalar potential is stable, or in other words at which scale we should expect new physics to emerge. ${ }^{1}$ The hierarchy problem associated to the Higgs boson mass has suggested that new physics should appear around the $\mathrm{TeV}$ scale. Since the most promising extension of the SM to address the hierarchy problem is supersymmetry (SUSY), we expected that the stop or the gluino should be around the corner. However the first searches up to $\sim 5 \mathrm{fb}^{-1}$ at the $\mathrm{LHC}^{2}$ have pushed the bounds on squark and gluino masses beyond the $\mathrm{TeV}$ scale. Even if the analysis have been performed within specific frameworks, such as Constrained Minimal Supersymmetric Standard Model (CMSSM) or minimal super-gravity (MSUGRA), what the most recent results suggest is that if SUSY exists one probably should be open minded as to how exactly it is realized. Open issues in this regard are the precise mechanism of SUSY breaking and whether Rparity is conserved. Indeed, supersymmetry may well be broken by a non-gravitational messenger. Similarly, one can have supersymmetry without R-parity [4-6]. Hence the need

\footnotetext{
${ }^{1}$ See e.g. [3].

${ }^{2}$ See e.g. talk by E. Halkiadakis for the CMS collaboration, January 312012.
} 
to consider alternative scenarios [7] where, in addition, the stringent bounds on the squark and gluino masses are relaxed $[8,9]$.

Apart from stabilizing the Higgs boson scalar potential, supersymmetry could address other Standard Model puzzles for which new physics is invoked. Among these we have that supersymmetry might explain the origin of neutrino masses as well as cold dark matter. Regarding the latter it has recently been shown that a relatively light gravitino in the few $\mathrm{GeV}$ range can provide a perfectly valid and interesting alternative in broken R-parity models [10]. Moreover, it provides a testable minimal mechanism for the origin of neutrino masses [11]. Regarding neutrinos it is well-known that bilinear R-parity violation offers a simple way to generate neutrino masses in supersymmetry [11]. In its "generic" formulation the model can not address issues associated to fermion mass hierarchies and mixings, such as those of neutrinos. Both Abelian [12-17] and non-Abelian [18-22] flavor symmetries have been used in the literature to constrain the R-parity violating terms. In this letter we propose a flavored version of bilinear $\mathrm{R}$-parity violation. The model has a single supersymmetric R-parity violating parameter allowed by the flavor symmetry $A_{4} \times Z_{2}$, where $A_{4}$ is the group of even permutations of four objects. This R-parity violating term is used to generate neutrino masses as required by current oscillation data, see [23]. We obtain predictions for the charged fermion masses as well as for neutrinos.

The paper is organized as follows: in section 2 we briefly review neutrino mass generation through low-scale supersymmetry with bilinear R-parity violation [24] and in section 3 we extend it by implementing a discrete flavor symmetry. In section 4 we present our results in the neutrino sector, where we find a correlation between the lepton mixing angles $\theta_{13}$ and $\theta_{23}$. In section 5 we comment on the scalar potential and finally in section 6 we summarize the main predictions of the model.

\section{Bilinear R-parity violation}

Bilinear R-parity Violation [4, 24] is the minimal extension of the Minimal Supersymmetric Standard Model (MSSM) that incorporates lepton number violation, providing a simple way to accommodate neutrino masses in supersymmetry. The superpotential is

$$
W=W^{\mathrm{MSSM}}+\epsilon_{i} \widehat{L}_{i} \widehat{H}_{u} .
$$

The three $\epsilon_{i}=\left(\epsilon_{e}, \epsilon_{\mu}, \epsilon_{\tau}\right)$ parameters have dimensions of mass and explicitly break lepton number. Their origin (and size) can be naturally explained in extended models where the breaking of lepton number is spontaneous [25-27]. In that sense, BRPV can be seen as an effective description of a more general supersymmetric framework for lepton number violation. In any case, the $\epsilon_{i}$ parameters are constrained to be small $\left(\epsilon_{i} \ll m_{W}\right)$ in order to account for the small neutrino masses. Furthermore, the presence of the new superpotential terms implies new soft SUSY breaking terms as well

$$
V_{\text {soft }}^{b-\mathbb{R}_{p}}=B_{\epsilon_{i}} \tilde{L}_{i} H_{u}
$$

where the $B_{\epsilon_{i}}$ parameters have dimensions of mass squared. The $\epsilon_{i}$ and $B_{\epsilon_{i}}$ couplings induce vacuum expectation values (VEVs) for the sneutrinos, $\left\langle\tilde{\nu}_{L}\right\rangle \equiv v_{L_{i}}$, proportional to the $\epsilon_{i}$, hence small, as required (we assume $B_{\epsilon_{i}}=B \epsilon_{i}$ ). 
In the presence of BRPV couplings, neutrinos and neutralinos mix, giving rise to neutrino masses [28-30]. In the basis $\left(\psi^{0}\right)^{T}=\left(-i \tilde{B}^{0},-i \tilde{W}_{3}^{0}, \widetilde{H}_{d}^{0}, \widetilde{H}_{u}^{0}, \nu_{e}, \nu_{\mu}, \nu_{\tau}\right)$ the neutral fermion mass matrix $M_{N}$ is given by

$$
M_{N}=\left(\begin{array}{cc}
\mathcal{M}_{\chi^{0}} & m^{T} \\
m & 0
\end{array}\right),
$$

where $\mathcal{M}_{\chi^{0}}$ is the usual neutralino mass matrix and

$$
m=\left(\begin{array}{cccc}
-\frac{1}{2} g^{\prime} v_{L_{e}} & \frac{1}{2} g v_{L_{e}} & 0 & \epsilon_{e} \\
-\frac{1}{2} g^{\prime} v_{L_{\mu}} & \frac{1}{2} g v_{L_{\mu}} & 0 & \epsilon_{\mu} \\
-\frac{1}{2} g^{\prime} v_{L_{\tau}} & \frac{1}{2} g v_{L_{\tau}} & 0 & \epsilon_{\tau}
\end{array}\right),
$$

is the matrix that characterizes the breaking of R-parity. Note that its elements are suppressed with respect to those in $\mathcal{M}_{\chi^{0}}$ due to the smallness of the $\epsilon_{i}$ parameters. Therefore, the resulting $M_{N}$ matrix has a type-I seesaw structure and the effective light neutrino mass matrix can be obtained with the usual formula $m_{\nu}^{0}=-m \cdot \mathcal{M}_{\chi^{0}}^{-1} \cdot m^{T}$, which can be expanded to give

$$
\left(m_{\nu}^{0}\right)_{i j}=a^{(0)} \Lambda_{i} \Lambda_{j}
$$

where $a^{(0)}$ is a combination of SUSY parameters and

$$
\Lambda_{i}=\mu v_{i}+v_{d} \epsilon_{i}
$$

are the so-called alignment parameters. The projective form of $m_{\nu}^{0}$ implies only one eigenvalue is non-zero. A natural choice is to ascribe this eigenvalue to the atmospheric scale. In this case the required solar mass scale, $\Delta m_{\text {sol }}^{2} \ll \Delta m_{\text {atm }}^{2}$, arises radiatively, at the 1-loop level, correcting the tree-level neutrino mass matrix in eq. (2.5). Detailed computations of the 1-loop contributions to the neutrino mass matrix can be found in refs. [28, 29]. The corrections are of the type

$$
\left(m_{\nu}^{1}\right)_{i j} \approx a^{(1)} \Lambda_{i} \Lambda_{j}+b^{(1)}\left(\Lambda_{i} \epsilon_{j}+\Lambda_{j} \epsilon_{i}\right)+c^{(1)} \epsilon_{i} \epsilon_{j}
$$

where the coefficients $a^{(1)}, b^{(1)}, c^{(1)}$ are complicated functions of the SUSY parameters. This generates a second non-zero mass eigenstate associated with the solar scale, and the corresponding mixing angle $\theta_{12}$. Note that the neutrino mixing angles are determined as ratios of $\not_{p}$ parameters $\epsilon_{i}$ and $\Lambda_{i}$.

Let us say a few words about the phenomenology of BRPV. The breaking of R-parity has an immediate consequence at colliders: the LSP in no longer stable and decays typically inside the detectors. Since LSP decays and neutrino masses have a common origin, one can show that ratios of LSP decay branching ratios correlate with the neutrino mixing angles measured at low energies [31-34]. This establishes a tight link which allows one to use neutrino oscillation data to test the model at the LHC see e. g. [35]. 


\begin{tabular}{|c|c|c|c|c|c|c|c|c|}
\hline & $\hat{Q}$ & $\hat{u}^{c}$ & $\hat{d}^{c}$ & $\hat{L}$ & $\hat{e}^{c}$ & $\hat{H}_{u}$ & $\hat{H}_{d}$ & $\hat{S}$ \\
\hline$A_{4}$ & 3 & 3 & 3 & 3 & 3 & 3 & 3 & 1 \\
\hline$Z_{2}$ & + & - & + & - & - & - & + & - \\
\hline
\end{tabular}

Table 1. The model assignments.

\section{The flavored BRpV model}

Let us consider the MSSM particle content extended with one extra singlet superfield $\hat{S}$ and a $A_{4} \times Z_{2}$ flavor symmetry with the assignments given in table 1 . The superfield $\hat{S}$ is required in order to generate the $\mu$ term, and is the only singlet under $A_{4}$. On the other hand the $Z_{2}$ symmetry forbids all $\mathbb{R}_{p}$ operators with the only exception of the bilinear terms $L H_{u}$, while the quark and charged lepton sectors are very similar to those in [36]. The assumption that all matter fields as well as the up and down Higgs doublets are in triplet representations of $A_{4}$ reduces the different BRPV parameters to only one.

The superpotential of the model is

$$
\mathcal{W}=Y\left(\widehat{L} \widehat{e}^{c}\right)_{3} \widehat{H_{d}}+\epsilon \widehat{L} \widehat{H_{u}}+\lambda \widehat{H_{u}} \widehat{H_{d}} \hat{S}+m_{S} \hat{S} \hat{S} .
$$

Note that, due to the product rule $3 \times 3=1+1^{\prime}+1^{\prime \prime}+3_{1}+3_{2}$, where $1,1^{\prime}, 1^{\prime \prime}$ are different singlets of $A_{4}$ and $3_{1,2}$ are different triplets, the assignment in table 1 allows for two different contractions in the usual charged lepton Yukawa interactions, compactly denoted by the first term in eq. (3.1). This leads to the couplings $Y_{\delta}\left|\epsilon_{i j k}^{\delta}\right| L_{i} e_{j}^{c} H_{d_{k}}$ with $\delta=1,2$. For $\delta=1(i j k)=(123),(231),(312)$ and for $\delta=2(i j k)=(213),(321),(132)$. The resulting quarks and charged lepton mass matrices have the form $[36,37]$

$$
M_{f}=\left(\begin{array}{ccc}
0 & y_{1}^{f}\left\langle H_{3}^{f}\right\rangle & y_{2}^{f}\left\langle H_{2}^{f}\right\rangle \\
y_{2}^{f}\left\langle H_{3}^{f}\right\rangle & 0 & y_{1}^{f}\left\langle H_{1}^{f}\right\rangle \\
y_{1}^{f}\left\langle H_{2}^{f}\right\rangle & y_{2}^{f}\left\langle H_{1}^{f}\right\rangle & 0
\end{array}\right), \quad f=u, d, l,
$$

where $d$-type quarks and charged fermions $l$ couple to the same Higgs. Note that since all matter fields and Higgs scalars are in triplets of $A_{4}$, the diagonal elements of the charged fermion mass matrices vanish. With the VEV alignment ${ }^{3}$

$$
\begin{aligned}
& \left\langle H_{u}\right\rangle=\frac{1}{\sqrt{2}}\left(v_{u_{1}}, v_{u_{2}}, v_{u_{3}}\right)=v_{u_{3}}\left(r^{u},-1,1\right) \\
& \left\langle H_{d}\right\rangle=\frac{1}{\sqrt{2}}\left(v_{d_{1}}, v_{d_{2}}, v_{d_{3}}\right)=v_{d_{3}}\left(r^{d},-1,1\right) \\
& \left\langle\tilde{\nu}_{L}\right\rangle=\frac{1}{\sqrt{2}}\left(v_{L_{e}}, v_{L_{\mu}}, v_{L_{\tau}}\right)=v_{L_{\tau}}\left(a^{\nu},-1,1\right),
\end{aligned}
$$

\footnotetext{
${ }^{3}$ This VEV alignment will be justified in section 5 , where it will be explicitly shown to be consistent with the minimization of the scalar potential of our model. However, note that the equality of the second and third entries in eqs. (3.3)-(3.5) is an assumption required to obtain phenomenologically acceptable fermion mass matrices.
} 
we have in the charged fermion sector nine parameters, one of which can be reabsorbed. These are used to fit nine masses and three mixing angles, hence four predictions emerge [36], given below as eqs. (3.11) and (3.12), in addition to $V_{u b}=0=V_{c b}$. At this stage we have unmixed leptons and $\mathrm{CP}$ conserved in the quark sector. Small nonzero $V_{u b}, V_{c b}$ can arise by mixing with vector-like quarks [38]. ${ }^{4}$ To see this in more detail, let us rewrite the fermion mass matrix in eq. (3.2). With the VEV alignments in eqs. (3.3)-(3.4), the mass matrix in eq. (3.2) can be rewriten as

$$
M_{f}=\left(\begin{array}{ccc}
0 & a^{f} & -b^{f} \\
b^{f} & 0 & a^{f} r^{f} \\
-a^{f} & b^{f} r^{f} & 0
\end{array}\right),
$$

where $a^{f}=y_{1}^{f} v_{3}^{f}, b=y_{2}^{f} v_{3}^{f}$. From eq. (3.6) we can see the mass matrix for the charged fermions has only three free parameters which can be written as functions of the charged fermion masses. Now we can consider the squared mass matrix for the charged fermions, $M_{f} M_{f}^{T}$

$$
M_{f} M_{f}^{T} \approx\left(\begin{array}{ccc}
\left(b^{f}\right)^{2} & -a^{f} b^{f} r^{f} & a^{f} b^{f} r^{f} \\
-a^{f} b^{f} r^{f} & \left(a^{f} r^{f}\right)^{2} & -a^{f} b^{f} \\
a^{f} b^{f} r^{f} & -a^{f} b^{f} & \left(b^{f} r_{f}\right)^{2}
\end{array}\right)
$$

where we have assumed $a^{f} \ll b^{f} \ll r^{f}$ (see below). The invariants of this matrix give rise to three equations in terms of the fermion masses. From these one can find the parameters, $a^{f}, b^{f}$ and $r^{f}$ as funtions of the charged fermion masses as [36, 37]

$$
\begin{aligned}
a^{f} & \approx \frac{m_{2}^{f}}{m_{3}^{f}} \sqrt{m_{1}^{f} m_{2}^{f}} \\
b^{f} & \approx \sqrt{m_{1}^{f} m_{2}^{f}} \\
r^{f} & \approx \frac{m_{3}^{f}}{\sqrt{m_{1}^{f} m_{2}^{f}}} .
\end{aligned}
$$

From eq. (3.10) we have the first prediction of the model, a quark-lepton mass relation:

$$
\frac{m_{\tau}}{\sqrt{m_{e} m_{\mu}}} \approx \frac{m_{b}}{\sqrt{m_{d} m_{s}}}
$$

due to the equality $r^{d}=r^{l}$. As discussed in ref. [36] such a formula works very well experimentally and, in contrast to the well-known Georgi-Jarlskog relation, does not arise from Clebsch Gordan coefficients, but follows simply from the equality of the two functions $r^{d}\left(m_{d}, m_{s}, m_{b}\right)=r^{l}\left(m_{e}, m_{\mu}, m_{\tau}\right)$. Moreover, it involves mass ratios, instead of absolute masses, hence more stable from the renormalization viewpoint.

\footnotetext{
${ }^{4}$ Vector-like quarks and their phenomenology have been widely studied in the literature. See for example the recent refs. [39, 40].
} 
The second prediction is the Cabibbo angle, which follows from the fact that the matrix in eq. (3.7) is diagonalized by [37]

$$
U_{f} \approx\left(\begin{array}{ccc}
1 & \sqrt{\frac{m_{1}^{f}}{m_{2}^{f}}} & 0 \\
-\sqrt{\frac{m_{1}^{f}}{m_{2}^{f}}} & 1 & 0 \\
0 & 0 & 1
\end{array}\right) .
$$

This formula is simply the well-known Gatto-Sartori-Tonin relation [41]. Indeed from the matrix in eq. (3.7) one obtains for instance the $V_{12}$ mixing as $V_{12} \sim a^{f} /\left(b^{f} r^{f}\right) \sim \sqrt{m_{1}^{f} / m_{2}^{f}}$ which gives the famous Gatto-Sartori-Tonin relation.

Let us now turn to the neutrino sector. As already discussed in the previous section, the tree-level neutrino mass matrix in eq. (2.5) has rank one. However it is straightforward to show that with the VEV alignment in eqs. (3.3), (3.4) and (3.5) we have

$$
\Lambda_{\mu}=-\Lambda_{\tau}
$$

where the $\Lambda_{i}$ defined in (2.6) now take the form

$$
\Lambda_{i}=\mu v_{L_{i}}+v_{d_{i}} \epsilon
$$

with $\mu=\lambda\langle S\rangle=\lambda v_{s} / \sqrt{2}$. Note that the $\epsilon_{i} v_{d}$ contributions that characterize "generic" BRPV models described in the previous section, have now become $\epsilon v_{d_{i}}$, where $i=(1,2,3)$, with a single bilinear $\epsilon$ parameter, due to the flavor symmetry.

Once the 1-loop corrections are included we have,

$$
m_{\nu}=m_{\nu}^{0}+m_{\nu}^{1}
$$

Using eqs. (2.5) and (2.7) and imposing the VEV alignment in eqs. (3.3), (3.4) and (3.5), we find the resulting neutrino mass matrix

$$
m_{\nu}=\left(\begin{array}{ccc}
c+\alpha(2 b+\alpha a) & c+b(\alpha-1)-\alpha a b+c+\alpha(a+b) \\
c+b(\alpha-1)-\alpha a & a-2 b+c & c-a \\
b+c+\alpha(a+b) & c-a & a+2 b+c
\end{array}\right),
$$

where the following definitions have been made

$$
\begin{aligned}
\Lambda_{e} & =\alpha \Lambda \\
\Lambda_{\tau} & =-\Lambda_{\mu}=\Lambda \\
a & =\left(a^{(0)}+a^{(1)}\right) \Lambda^{2} \\
b & =b^{(1)} \Lambda \epsilon \\
c & =c^{(1)} \epsilon^{2}
\end{aligned}
$$

Note that the 1-loop contributions are dis-aligned with respect to the tree-level one, with the tree-level degeneracy lifted by radiative corrections. In the limit $\alpha=0$ the 
neutrino mass matrix has the massless eigenvector which corresponds to $(2,-1,-1)^{T}$, called tri-bimaximal-1 for instance in ref. [42]. In the limit of $\alpha=b=0$ the spectrum is tri-bimaximal.

Finally, in the limit $b=c=0$ one recovers the tree-level mass matrix $m_{\nu}^{0}$. This matrix has rank one, and thus only one eigenvalue is non-zero, $m_{\nu_{3}}=a|\vec{\Lambda}|^{2}=a\left(2+\alpha^{2}\right)$. The associated eigenvector lies along the direction $(\alpha, 1,-1)$. Although there are corrections from the charged lepton sector and from the 1-loop contributions, the condition $|\alpha| \ll 1$ ensures a small $\theta_{13}$ value. ${ }^{5}$ Similarly, one expects the hierarchy $b, c \ll a$, since $b$ and $c$ are generated at the 1-loop level, whereas $a$ is a tree-level parameter. This naturally implies $m_{\nu_{2}} \ll m_{\nu_{3}}$.

In conclusion, the neutrino mass spectrum is compatible with normal hierarchy, with a radiatively induced solar scale. The solar and atmospheric mass square differences as well as the solar mixing angle can be fitted as shown explicitly in ref. [28].

\section{Large $\theta_{13}$ and deviations from maximal atmospheric mixing}

In the CP-conserving case, the neutrino mass matrix in eq. (3.15) is characterized by 4 free parameters, for 6 observables in total, three masses and three mixing angles, therefore two predictions can be obtained. ${ }^{6}$ The first one is the mass of the lightest neutrino, $m_{\nu_{1}}=0$, since the matrix in eq. (3.15) has a null eigenvalue (this state gets a negligibly tiny mass once 2-loop contributions are included). The second prediction is a correlation among the neutrino mixing angles, which we determine for the recent global fits taking into account the latest experimental data presented at the recent Neutrino 2012 conference. The results of our analysis of the parameter space corresponding to the global oscillation fits in Forero et al [43], Fogli et al [44] and Gonzalez-Garcia et al [45] $]^{7}$ are given in figures 1,2 , and 3, respectively.

From the presented correlations between the atmospheric and solar mixing angle, as well as the resulting allowed ranges of the reactor and atmospheric angle, it is clear that our model restricts the oscillation parameters in a non-trivial way, however consistent with the $1 \sigma$ ranges for the neutrino oscillation parameters given by all global fits, in particular with the "large" reactor mixing angle and non-maximal atmospheric mixing hinted by the most recent oscillation data. ${ }^{8}$ At $2 \sigma$, predictions in our BRPV model become very weak.

\section{Scalar potential and spectrum}

The presence of additional Higgs doublets is a common feature to many flavor models and leads to a complicated structure of the scalar potential. Therefore, though non-trivial, the

\footnotetext{
${ }^{5}$ In our numerical analysis we have found that $|\alpha| \sim 0.2$ leads to $\theta_{13}$ in the observed range.

${ }^{6}$ Note that neutrino mixing receives additional corrections from the charged lepton sector. However, these do not involve any additional parameter. Although relatively small, they have been taken into account in our numerical analysis.

${ }^{7}$ See also previous analyses in $[46,47]$.

${ }^{8}$ The main difference is the presence of two allowed octants for the atmospheric mixing in the analyses of $[43,45-47]$ while only one octant is present in the Bari group analysis, since it is more strongly preferred in that case [44].
} 

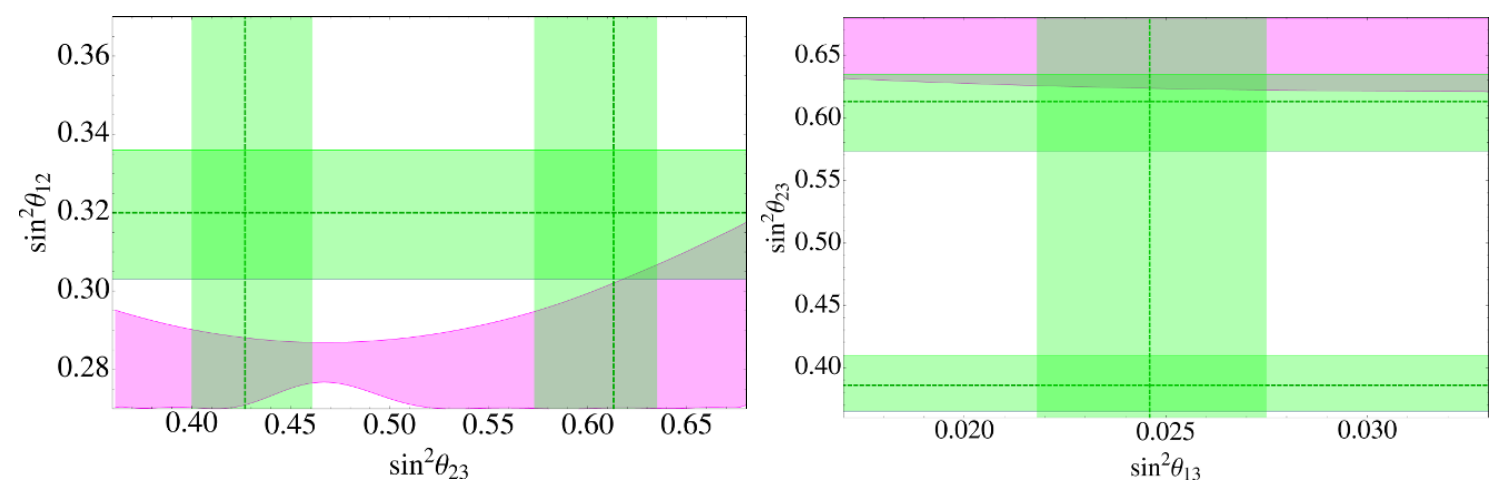

Figure 1. Left: predicted correlation between the atmospheric and solar mixing angle taking the $3 \sigma$ oscillation parameter ranges from the global fit of ref. [43]. The dashed lines represent the best-fit while the green and blue bands correspond to the $1 \sigma$ range. As can be seen, there is a region consistent at $1 \sigma$ with the global fit of oscillation parameters. Right: the allowed range of reactor and atmospheric mixing angles, taking the $1 \sigma$ range for the solar mixing angle. The dashed lines correspond to the best fit values, while the straight bands correspond to the $1 \sigma$ ranges. As can be seen, maximal atmospheric mixing is excluded at $1 \sigma$.
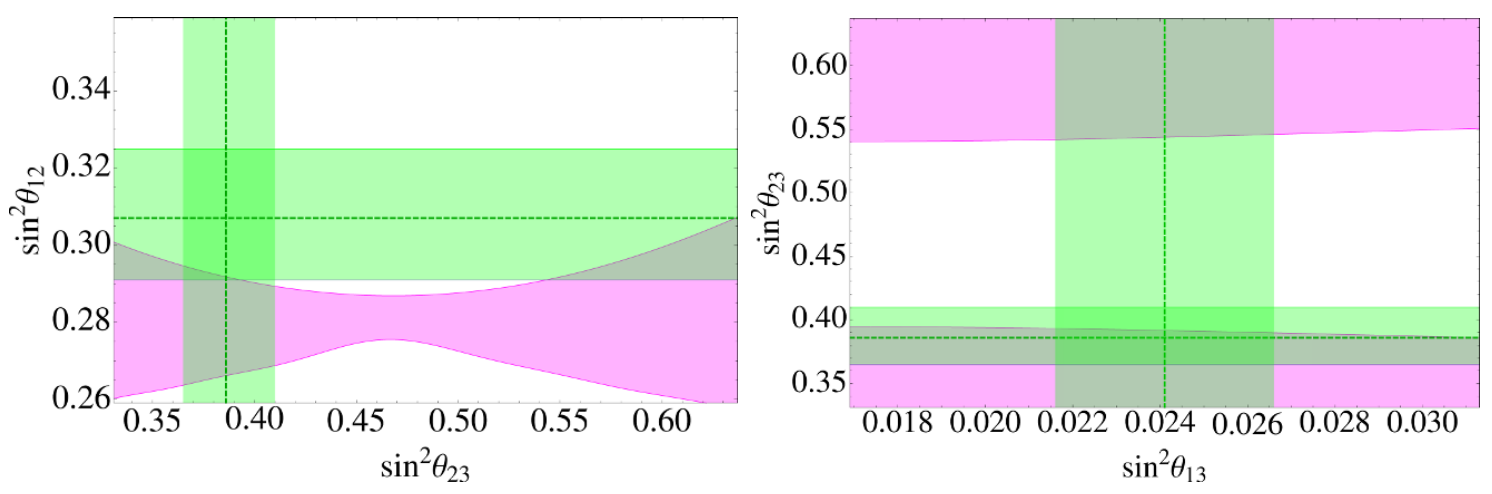

Figure 2. Same as figure 1 for the analysis in ref. [44].
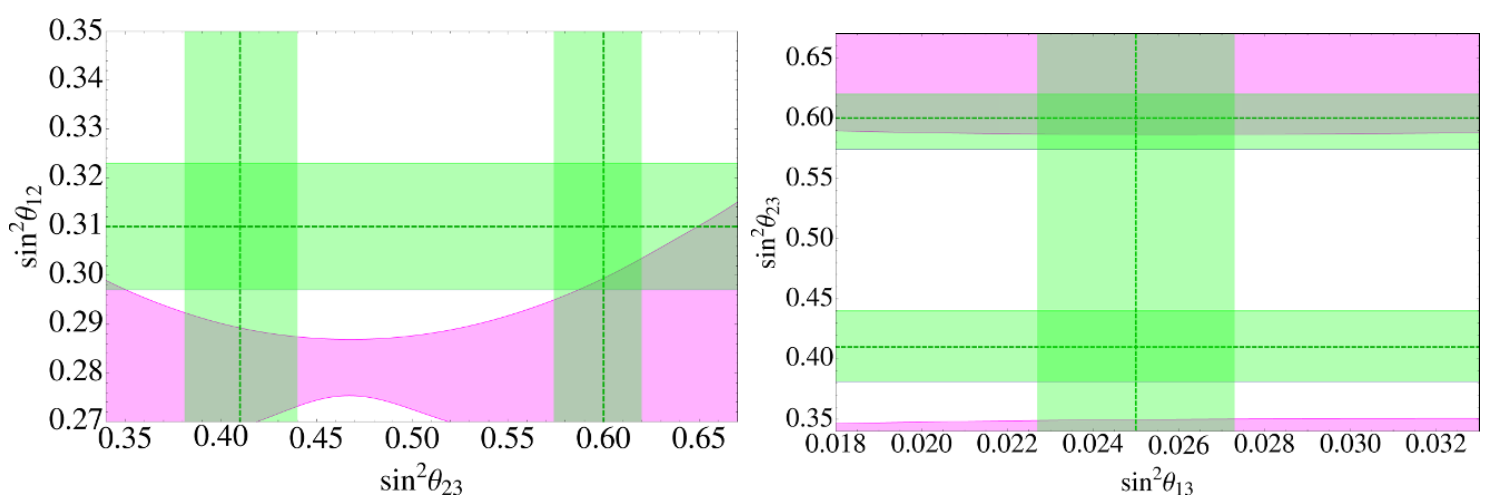

Figure 3. Same as figure 1 for the analysis in ref. [45-47]. 
determination of a phenomenologically viable minimum of the potential is of fundamental importance. The scalar potential can be split as

$$
V=V_{F}+V_{D}+V_{\text {soft }}^{A_{4}}+V_{\text {soft }}^{A_{4}}
$$

where $V_{F}$ and $V_{D}$ are the usual F- and D-terms and $V_{\text {soft }}^{A_{4}}$, defined as

$$
\begin{aligned}
V_{\mathrm{soft}}^{A_{4}}= & T_{Y_{1}}\left(\tilde{L} \tilde{e}^{*}\right)_{3_{1}} H_{d}+T_{Y_{2}}\left(\tilde{L} \tilde{e}^{*}\right)_{3_{2}} H_{d}+T_{\lambda} H_{d} H_{u} S \\
& +B_{\epsilon} \tilde{L} H_{u}-B_{m_{S}} S^{2}+m_{s}^{2}|S|^{2}+\text { h.c. }
\end{aligned}
$$

contains soft SUSY breaking terms that preserve the flavor symmetry. ${ }^{9}$ In addition, we allow for the existence of an additional piece, $V_{\text {soft }}^{A_{4}}$, with terms that break softly both SUSY and $A_{4}$ but preserve $Z_{2}$. The presence of this piece is necessary in order to obtain a realistic spectrum. The $A_{4} \times Z_{2}$ flavor symmetry leads to accidental continuous symmetries in the scalar potential which, after they get spontaneously broken by the corresponding VEVs, imply the existence of additional Goldstone bosons. These massless states couple to the gauge bosons thus the explicit breaking of those continuous symmetries is required from a phenomenological point of view. ${ }^{10}$

We have considered the following soft breaking of $A_{4}$

$$
V_{\mathrm{soft}}^{A_{4}}=\sum_{i j} m_{H_{d_{i}} H_{d_{j}}}^{2} H_{d_{i}} H_{d_{j}}^{*}+\sum_{i j} m_{H_{u_{i}} H_{u_{j}}}^{2} H_{u_{i}} H_{u_{j}}^{*}+\sum_{i j} m_{l_{i} l_{j}}^{2} \tilde{L}_{i} \tilde{L}_{j}^{*}+\sum_{i j} m_{e_{i} e_{j}}^{2} \tilde{e}_{i} \tilde{e}_{j}^{*}
$$

Note that $A_{4}$ would enforce $m_{H_{d_{i}} H_{d_{j}}}^{2}=m_{H_{u_{i}} H_{u_{j}}}^{2}=m_{l_{i} l_{j}}^{2}=m_{e_{i} e_{j}}^{2}=0$ for $i \neq j$ and the equality of the soft masses for all elements in the same $A_{4}$ triplet. In our analysis we have broken those relations explicitly and found minima of the scalar potential with realistic spectra in the scalar, pseudoscalar and charged scalar sectors. ${ }^{11}$

The minimization of the scalar potential must also lead to the required vacuum alignment, see equations (3.3), (3.4) and (3.5). That restricts the allowed parameter space. In particular, the conditions $\left|\alpha^{u}\right| \ll\left|r^{u}\right|$ and $\left|\alpha^{d}\right| \ll\left|r^{d}\right|$ can be naturally fulfilled with large soft masses for the second and third generations of Higgs doublets in the $(1-1000 \mathrm{TeV})^{2}$ range (see below). That can be easily seen from the tadpole equations. For example, for $v_{d_{2}}$ one finds

$$
\begin{gathered}
\frac{\partial V}{\partial v_{d_{2}}}=\frac{1}{8}\left(4 m_{H_{d_{1}} H_{d_{2}}}^{2} v_{d_{1}}+8 m_{H_{d_{2}}}^{2} v_{d_{2}}+4 m_{H_{d_{2}} H_{d_{3}}}^{2} v_{d_{3}}+\left(g_{1}^{2}+g_{2}^{2}\right)\left(\left|\vec{v}_{d}\right|^{2}-\left|\vec{v}_{u}\right|^{2}+\left|\vec{v}_{L}\right|^{2}\right) v_{d_{2}}\right. \\
+4|\lambda|^{2}\left(v_{d_{2}} v_{s}^{2}+v_{d_{2}} v_{u_{2}}^{2}+v_{d_{1}} v_{u_{1}} v_{u_{2}}+v_{d_{3}} v_{u_{2}} v_{u_{3}}\right)
\end{gathered}
$$

\footnotetext{
${ }^{9}$ For the sake of clarity we do not specify here the $A_{4}$ contractions. We also omit the soft gaugino masses, which of course preserve $A_{4} \times Z_{2}$.

${ }^{10}$ We do not attempt to provide a complete explanation about the origin of the terms in the scalar potential, nor about the hierarchy among them. On general grounds one expects that, for generic dynamics in the hidden sector responsible for supersymmetry breaking, the flavor symmetry is not respected. That is the reason why we allow for $A_{4}$ breaking terms in the soft SUSY breaking scalar potential.

${ }^{11}$ In principle, additional terms of the type $m_{l_{i} H_{u_{j}}}^{2}$ are allowed in $V_{\text {soft }}^{A_{4}}$. However, the presence of such terms would introduce additional sources of lepton number violation and destabilize the required vacuum alignment.
} 


$$
-4 \sqrt{2} v_{s}\left(2 v_{u_{2}} \operatorname{Re}\left(\lambda m_{S}^{*}\right)+v_{L_{2}} \operatorname{Re}\left(\lambda^{*} \epsilon\right)+v_{u_{2}} \operatorname{Re}\left(T_{\lambda}\right)\right)=0
$$

Here we have defined $m_{H_{d_{2}}}^{2} \equiv m_{H_{d_{2}} H_{d_{2}}}^{2},\left|\vec{v}_{d}\right|^{2} \equiv \sum_{i} v_{d_{i}}^{2},\left|\vec{v}_{u}\right|^{2} \equiv \sum_{i} v_{u_{i}}^{2}$ and $\left|\vec{v}_{L}\right|^{2} \equiv \sum_{i} v_{L_{i}}^{2}$. Neglecting small $R_{p}$ contributions and assuming the aforementioned VEV hierarchy and $\mathrm{CP}$ conservation, equation (5.4) can be solved to give the simple estimate

$$
v_{d_{2}} \simeq-\frac{4 v_{d_{1}} m_{H d_{1} H d_{2}}^{2}}{\left(g_{1}^{2}+g_{2}^{2}\right)\left(v_{d_{1}}^{2}-v_{u_{1}}^{2}\right)+8 m_{H_{d_{2}}}^{2}-4 m_{H d_{2} H d_{3}}^{2}+4 \lambda^{2} v_{s}^{2}} \approx \frac{\sqrt{m_{d} m_{s}}}{m_{b}} v_{d_{1}},
$$

where the last equality is obtained from eqs. (3.4) and (3.10). Thus $\left|v_{d_{2}}\right| \ll\left|v_{d_{1}}\right|$ is obtained if $m_{H_{d_{2}}}^{2} \gg m_{H_{d_{1} H_{d_{2}}}}^{2}, m_{H_{d_{2}} H_{d_{3}}}^{2} \sim m_{\mathrm{SUSY}}^{2}$. Similar tadpole equations can be found for $v_{d_{3}}$ and $v_{u_{2,3}}$, leading to analogous hierarchies for the corresponding soft squared masses. ${ }^{12}$ Finally, as discussed in section 2, the sneutrino VEVs are naturally small since these "induced VEVs" are proportional to the $\epsilon$ parameter. This can be seen in the corresponding tadpole equations,

$$
\begin{aligned}
\frac{\partial V}{\partial v_{L_{i}}}=\frac{1}{8} & \left(8 \operatorname{Re}\left(B_{\epsilon}\right) v_{u_{i}}+v_{L_{i}}\left(g_{1}^{2}+g_{2}^{2}\right)\left(\left|\vec{v}_{d}\right|^{2}-\left|\vec{v}_{u}\right|^{2}+\left|\vec{v}_{L}\right|^{2}\right)\right. \\
& \left.+4\left(m_{l_{i} l_{i}}^{2} v_{L_{i}}+\sum_{j} m_{l_{i} l_{j}}^{2} v_{L_{j}}\right)+8 v_{L_{i}}|\epsilon|^{2}-4 \sqrt{2} v_{d_{i}} v_{s} \operatorname{Re}\left(\epsilon \lambda^{*}\right)\right)
\end{aligned}
$$

which imply that all $v_{L_{i}}$ vanish in the $\epsilon=B_{\epsilon}=0$ limit.

The requirement of very large soft squared masses has important consequences on the mass spectrum. Dominated by the contributions from the soft terms, the spectrum contains several degenerate $\left\{H^{0}, A^{0}, H^{ \pm}\right\}$sets, some of them with masses in the $10-100 \mathrm{TeV}$ range. This degeneracy is very strong in the case of $H^{0}$ and $A^{0}$ and only slightly broken for $H^{ \pm}$due to its mixing with the charged sleptons.

Another interesting feature of the spectrum is the decrease of the mass of the scalar singlet for increasing $\tan \beta$, where

$$
\tan \beta=\frac{v_{u_{1}}}{v_{d_{1}}}
$$

In fact, for $\tan \beta \sim 10$ one can easily find points in parameter space with a light scalar singlet, $S_{1}$, in the range of $100 \mathrm{MeV}-20 \mathrm{GeV}$. This result can be easily understood in a simple limit. Neglecting the mixing with the doublet states, the leading contribution to the squared mass of the scalar singlet is

$$
m_{S_{1}}^{2}=\frac{\tan \beta v^{2}\left(2 \lambda m_{S}+T_{\lambda}\right)}{\sqrt{2}\left(1+\tan ^{2} \beta\right) v_{s}}
$$

where $v^{2}=(246 \mathrm{GeV})^{2}=4 m_{W}^{2} / g^{2}$ is the usual electroweak VEV. Eq. (5.8) shows that $m_{S_{1}}^{2}$ scales as $1 / \tan \beta$ for sufficiently large $\tan \beta$. This naturally leads to $m_{S_{1}}^{2} \ll v^{2}$ for $\tan \beta \gtrsim 10$. In such scenarios the Higgs decay channel $h \rightarrow S_{1} S_{1}$ can dominate the Higgs decay if the $h-S_{1}-S_{1}$ coupling is large. After the first hints of the existence of the Higgs

\footnotetext{
${ }^{12}$ In fact, the hierarchy is stronger in the $H_{u}$ sector since the ratio $v_{u_{2,3}} / v_{u_{1}}$ must be smaller in order to explain the mass hierarchy between the top quark mass and the charm and up quark masses.
} 
boson, later confirmed in refs. [48, 49], it has been increasingly clear that the properties of the discovered particle are very close to those of a SM Higgs boson [50-53]. Although there is still room for new interactions [54], these are now constrained by the data. This imposes an important restriction on the size of our $\lambda$ coupling, $\lambda \ll 1$.

As a generic example to illustrate these properties, we provide the following parameter set and results for a particular but generic point in parameter space.

\section{Parameters set.}

- Superpotential and $V_{\text {soft }}^{A_{4}}$ parameters: $\tan \beta=30, \lambda=0.01, m_{S}=88 \mathrm{TeV}, T_{\lambda}=$ $-2.3 \mathrm{TeV}, B_{m_{S}}=-0.79 \mathrm{TeV}^{2}, B_{\epsilon}=-4.25 \mathrm{TeV}^{2}$ and $m_{s}^{2}=-31000 \mathrm{TeV}^{2}$.

- $V_{\text {soft }}^{A_{4}}$ parameters:

$$
\begin{aligned}
& m_{H_{d_{i}} H_{d_{j}}}^{2}=\left(\begin{array}{rcc}
4.4 & -9.9 & 2.2 \\
500.0 & 5.1 \\
& 111.7
\end{array}\right) \mathrm{TeV}^{2} \\
& m_{H_{u_{i}} H_{u_{j}}}^{2}=\left(\begin{array}{ccc}
-0.003 & -7.6 & 6.2 \\
& 37900.0 & -8.6 \\
& & 31200.0
\end{array}\right) \mathrm{TeV}^{2} \\
& m_{l_{i} l_{j}}^{2}=\left(\begin{array}{rrc}
6800000.0 & 2.2 & -2.6 \\
& 62200.0 & -9.6 \\
& 8300.0
\end{array}\right) \mathrm{TeV}^{2}
\end{aligned}
$$

- Neutrino physics can be accommodated with $\epsilon \sim 10^{-4} \mathrm{GeV}$, which in turn results in $v_{L_{i}}$ of the same order. Similar soft terms are given in the charged scalar sector. In this parameter point one finds the VEV configuration: $v_{d_{1}}^{2}+v_{u_{1}}^{2} \simeq v^{2}$, $v_{d_{2}}=-v_{d_{3}} \sim 10^{-2} v_{d_{1}}, v_{u_{2}}=-v_{u_{3}} \sim 10^{-4} v_{u_{1}}$, and $v_{s}=-8 \mathrm{TeV}$.

\section{Results in the scalar sector.}

- A light scalar, $m_{S S}=120 \mathrm{MeV}$, of singlet nature with tiny $\left(\sim 10^{-8} \%\right)$ doublet admixture.

- A light scalar, $m_{h^{0}}=90.4 \mathrm{GeV}$, of doublet nature. This state can be identified with the Higgs boson. ${ }^{13}$

- Degeneracies between real and imaginary components of the sneutrinos.

- Very heavy (and degenerate) states with masses $m_{H^{0}} \simeq m_{A^{0}} \simeq m_{H^{ \pm}}$in the multi- $\mathrm{TeV}$ range.

\footnotetext{
${ }^{13}$ For simplicity we give tree-level results. Large 1-loop corrections are of course expected and bring the mass of the light doublet state to experimentally acceptable levels.
} 
With this generic prediction for the spectrum in the extended scalar sector the model turn outs to be safe from the constraints on the oblique $\mathrm{S}, \mathrm{T}, \mathrm{U}$ parameters. Besides the heaviness of some states, the degeneracies among their masses cancel their contributions to these precision observables. Note also that in our scenario the large supersymmetry breaking scale is related to the flavor symmetry used to get the required vaccuum alignment.

\section{Discussion and conclusions}

We have extended the (next to) MSSM by implementing a discrete non-Abelian flavor symmetry $A_{4} \times Z_{2}$. The most general renormalizable allowed superpotential forbids all the trilinear RPV terms (including those violating baryon number) and has a single bilinear R-parity violating term. Three copies of up and down Higgs doublets are required, in addition to a $\mathrm{SU}(3) \times \mathrm{SU}(2) \times \mathrm{U}(1)$ singlet present in $\hat{S}$, odd under $Z_{2}$. When these develop VEVs both the electroweak and flavor symmetries are broken and, in addition, sneutrinos acquire tiny VEVs. The Higgs fields align so as to recover the correct charged fermion mass hierarchies and the two successful predictions eqs. (3.11) and (3.12) [36].

As in the usual flavor-less BRPV model, the 1-loop radiative corrections is misaligned with the tree-level ones. These 1-loop contributions provide the solar mass square splitting. Due to the flavor symmetry and vacuum alignment, one neutrino is nearly massles and there is a non-trivial restriction upon the neutrino oscillation parameters, displayed in figures 1-3, consistent however with the most recent experimental data presented at the recent Neutrino 2012 conference and the corresponding neutrino oscillation global fits [43-47]. As far as collider physics is concerned, our model predicts that LSP decays and neutrino mixing angles are tightly correlated, opening encourageing expectations for searches at the LHC [35]. Finally, even though the usual neutralino LSP is lost as dark matter candidate, one can show that a relatively light gravitino provides a very interesting alternative [10].

\section{Acknowledgments}

We thank Martin Hirsch for useful discussions. Work supported by the Spanish MEC under grants FPA2008-00319/FPA, FPA2011-22975 and MULTIDARK CSD2009-00064 (Consolider-Ingenio 2010 Programme), by Prometeo/2009/091 (Generalitat Valenciana), by the EU ITN UNILHC PITN-GA-2009-237920. S. M. is supported by a Juan de la Cierva contract, E. P. by CONACyT (Mexico) and A.V. by the ANR project CPV-LFV-LHC NT09-508531.

Open Access. This article is distributed under the terms of the Creative Commons Attribution License which permits any use, distribution and reproduction in any medium, provided the original author(s) and source are credited.

\section{References}

[1] For the CMS collaboration, J. Incandela, Update on the Standard Model Higgs searches in CMS, CERN seminar, CERN, Geneva Switzerland July 42012. 
[2] For the ATLAS collaboration, F. Gianotti, Status of Standard Model Higgs searches in ATLAS, CERN seminar, CERN, Geneva Switzerland July 42012.

[3] J. Elias-Miro et al., Higgs mass implications on the stability of the electroweak vacuum, Phys. Lett. B 709 (2012) 222 [arXiv:1112.3022] [INSPIRE].

[4] L.J. Hall and M. Suzuki, Explicit R-parity breaking in supersymmetric models, Nucl. Phys. B 231 (1984) 419 [INSPIRE].

[5] G.G. Ross and J. Valle, Supersymmetric models without R-parity, Phys. Lett. B 151 (1985) 375 [INSPIRE].

[6] J.R. Ellis, G. Gelmini, C. Jarlskog, G.G. Ross and J. Valle, Phenomenology of supersymmetry with broken R-parity, Phys. Lett. B 150 (1985) 142 [INSPIRE].

[7] H. Dreiner, F. Staub, A. Vicente and W. Porod, General MSSM signatures at the LHC with and without R-parity, Phys. Rev. D 86 (2012) 035021 [arXiv:1205.0557] [INSPIRE].

[8] P.W. Graham, D.E. Kaplan, S. Rajendran and P. Saraswat, Displaced supersymmetry, JHEP 07 (2012) 149 [arXiv: 1204.6038] [INSPIRE].

[9] M. Hanussek and J. Kim, Testing neutrino masses in the R-parity violating minimal supersymmetric standard model with LHC results, Phys. Rev. D 85 (2012) 115021 [arXiv: 1205.0019] [INSPIRE].

[10] D. Restrepo, M. Taoso, J. Valle and O. Zapata, Gravitino dark matter and neutrino masses with bilinear R-parity violation, Phys. Rev. D 85 (2012) 023523 [arXiv:1109.0512] [INSPIRE].

[11] M. Hirsch and J.W.F. Valle, Supersymmetric origin of neutrino mass, New J. Phys. 6 (2004) 76 [hep-ph/0405015] [INSPIRE].

[12] V. Ben-Hamo and Y. Nir, Implications of horizontal symmetries on baryon number violation in supersymmetric models, Phys. Lett. B 339 (1994) 77 [hep-ph/9408315] [INSPIRE].

[13] T. Banks, Y. Grossman, E. Nardi and Y. Nir, Supersymmetry without R-parity and without lepton number, Phys. Rev. D 52 (1995) 5319 [hep-ph/9505248] [INSPIRE].

[14] P. Binetruy, E. Dudas, S. Lavignac and C.A. Savoy, Quark flavor conserving violations of the lepton number, Phys. Lett. B 422 (1998) 171 [hep-ph/9711517] [INSPIRE].

[15] J.R. Ellis, S. Lola and G.G. Ross, Hierarchies of R-violating interactions from family symmetries, Nucl. Phys. B 526 (1998) 115 [hep-ph/9803308] [INSPIRE].

[16] J. Mira, E. Nardi, D. Restrepo and J. Valle, Bilinear R-parity violation and small neutrino masses: a selfconsistent framework, Phys. Lett. B 492 (2000) 81 [hep-ph/0007266] [INSPIRE].

[17] G. Bhattacharyya, H. Pas and D. Pidt, R-parity violating flavor symmetries, recent neutrino data and absolute neutrino mass scale, Phys. Rev. D 84 (2011) 113009 [arXiv:1109.6183] [INSPIRE].

[18] C.D. Carone, L.J. Hall and H. Murayama, A supersymmetric theory of flavor and R-parity, Phys. Rev. D 54 (1996) 2328 [hep-ph/9602364] [INSPIRE].

[19] G. Bhattacharyya, Non-Abelian flavor symmetry and R-parity, Phys. Rev. D 57 (1998) 3944 [hep-ph/9707297] [INSPIRE]. 
[20] Y. Kajiyama, E. Itou and J. Kubo, Non-Abelian discrete family symmetry to soften the SUSY flavor problem and to suppress proton decay, Nucl. Phys. B $\mathbf{7 4 3}$ (2006) 74 [hep-ph/0511268] [INSPIRE].

[21] Y. Kajiyama, R-parity violation and non-Abelian discrete family symmetry, JHEP 04 (2007) 007 [hep-ph/0702056] [INSPIRE].

[22] Y. Daikoku and H. Okada, $S_{4} \times Z_{2}$ flavor symmetry in supersymmetric extra $\mathrm{U}(1)$ model, Phys. Rev. D 82 (2010) 033007 [arXiv:0910.3370] [inSPIRE].

[23] M. Maltoni, T. Schwetz, M. Tortola and J. Valle, Status of global fits to neutrino oscillations, New J. Phys. 6 (2004) 122 [hep-ph/0405172] [INSPIRE].

[24] M.A. Diaz, J.C. Romao and J. Valle, Minimal supergravity with R-parity breaking, Nucl. Phys. B 524 (1998) 23 [hep-ph/9706315] [INSPIRE].

[25] A. Masiero and J. Valle, A model for spontaneous R-parity breaking, Phys. Lett. B 251 (1990) 273 [INSPIRE].

[26] J. Romao, C. Santos and J. Valle, How to spontaneously break R-parity, Phys. Lett. B 288 (1992) 311 [INSPIRE].

[27] J. Romao, A. Ioannisian and J. Valle, Supersymmetric unification with radiative breaking of R-parity, Phys. Rev. D 55 (1997) 427 [hep-ph/9607401] [INSPIRE].

[28] M. Hirsch, M. Diaz, W. Porod, J. Romao and J. Valle, Neutrino masses and mixings from supersymmetry with bilinear $R$ parity violation: a theory for solar and atmospheric neutrino oscillations, Phys. Rev. D 62 (2000) 113008 [Erratum ibid. D 65 (2002) 119901] [hep-ph/0004115] [INSPIRE].

[29] M. Diaz, M. Hirsch, W. Porod, J. Romao and J. Valle, Solar neutrino masses and mixing from bilinear $R$ parity broken supersymmetry: analytical versus numerical results, Phys. Rev. D 68 (2003) 013009 [Erratum ibid. D 71 (2005) 059904] [hep-ph/0302021] [INSPIRE].

[30] E.J. Chun and S.K. Kang, One loop corrected neutrino masses and mixing in supersymmetric standard model without R-parity, Phys. Rev. D 61 (2000) 075012 [hep-ph/9909429] [INSPIRE].

[31] B. Mukhopadhyaya, S. Roy and F. Vissani, Correlation between neutrino oscillations and collider signals of supersymmetry in an R-parity violating model, Phys. Lett. B 443 (1998) 191 [hep-ph/9808265] [INSPIRE].

[32] S. Choi, E.J. Chun, S.K. Kang and J.S. Lee, Neutrino oscillations and R-parity violating collider signals, Phys. Rev. D 60 (1999) 075002 [hep-ph/9903465] [INSPIRE].

[33] J. Romao, M. Diaz, M. Hirsch, W. Porod and J. Valle, A supersymmetric solution to the solar and atmospheric neutrino problems, Phys. Rev. D 61 (2000) 071703 [hep-ph/9907499] [INSPIRE].

[34] W. Porod, M. Hirsch, J. Romao and J. Valle, Testing neutrino mixing at future collider experiments, Phys. Rev. D 63 (2001) 115004 [hep-ph/0011248] [INSPIRE].

[35] F. De Campos et al., Probing neutrino oscillations in supersymmetric models at the Large Hadron Collider, Phys. Rev. D 82 (2010) 075002 [arXiv: 1006.5075] [INSPIRE].

[36] S. Morisi, E. Peinado, Y. Shimizu and J. Valle, Relating quarks and leptons without grand-unification, Phys. Rev. D 84 (2011) 036003 [arXiv:1104.1633] [INSPIRE]. 
[37] S. Morisi and E. Peinado, An $A_{4}$ model for lepton masses and mixings, Phys. Rev. D 80 (2009) 113011 [arXiv:0910.4389] [INSPIRE].

[38] S. Morisi, M. Nebot, K.M. Patel, E. Peinado and J.W.F. Valle, in preparation.

[39] F. Botella, G. Branco and M. Nebot, The hunt for new physics in the flavour sector with up vector-like quarks, JHEP 12 (2012) 040 [arXiv:1207.4440] [INSPIRE].

[40] Y. Okada and L. Panizzi, LHC signatures of vector-like quarks, arXiv:1207.5607 [INSPIRE].

[41] R. Gatto, G. Sartori and M. Tonin, Weak selfmasses, Cabibbo angle and broken $\mathrm{SU}(2) \times \mathrm{SU}(2)$, Phys. Lett. B 28 (1968) 128 [INSPIRE].

[42] S. Antusch, S.F. King, C. Luhn and M. Spinrath, Trimaximal mixing with predicted $\theta_{13}$ from a new type of constrained sequential dominance, Nucl. Phys. B 856 (2012) 328 [arXiv: 1108.4278] [INSPIRE].

[43] D. Forero, M. Tortola and J. Valle, Global status of neutrino oscillation parameters after Neutrino-2012, Phys. Rev. D 86 (2012) 073012 [arXiv:1205.4018] [InSPIRE].

[44] G. Fogli et al., Global analysis of neutrino masses, mixings and phases: entering the era of leptonic CP-violation searches, Phys. Rev. D 86 (2012) 013012 [arXiv: 1205.5254] [INSPIRE].

[45] M. Gonzalez-Garcia, M. Maltoni, J. Salvado and T. Schwetz, Global fit to three neutrino mixing: critical look at present precision, arXiv:1209.3023 [INSPIRE].

[46] T. Schwetz, M. Tortola and J. Valle, Where we are on $\theta_{13}$ : addendum to 'global neutrino data and recent reactor fluxes: status of three-flavour oscillation parameters', New J. Phys. 13 (2011) 109401 [arXiv:1108.1376] [INSPIRE].

[47] T. Schwetz, M. Tortola and J. Valle, Global neutrino data and recent reactor fluxes: status of three-flavour oscillation parameters, New J. Phys. 13 (2011) 063004 [arXiv:1103.0734] [INSPIRE].

[48] CMS collaboration, Observation of a new boson at a mass of $125 \mathrm{GeV}$ with the CMS experiment at the LHC, Phys. Lett. B 716 (2012) 30 [arXiv:1207.7235] [INSPIRE].

[49] ATLAS collaboration, Observation of a new particle in the search for the Standard Model Higgs boson with the ATLAS detector at the LHC, Phys. Lett. B 716 (2012) 1 [arXiv: 1207.7214] [INSPIRE].

[50] T. Corbett, O. Eboli, J. Gonzalez-Fraile and M. Gonzalez-Garcia, Constraining anomalous Higgs interactions, Phys. Rev. D 86 (2012) 075013 [arXiv:1207.1344] [InSPIRE].

[51] J. Ellis and T. You, Global analysis of the Higgs candidate with mass $\sim 125 \mathrm{GeV}$, JHEP 09 (2012) 123 [arXiv: 1207.1693] [INSPIRE].

[52] D. Carmi, A. Falkowski, E. Kuflik, T. Volansky and J. Zupan, Higgs after the discovery: a status report, JHEP 10 (2012) 196 [arXiv:1207.1718] [INSPIRE].

[53] T. Plehn and M. Rauch, Higgs couplings after the discovery, Europhys. Lett. 100 (2012) 11002 [arXiv: 1207.6108] [INSPIRE].

[54] S. Banerjee, S. Mukhopadhyay and B. Mukhopadhyaya, New Higgs interactions and recent data from the LHC and the Tevatron, JHEP 10 (2012) 062 [arXiv:1207.3588] [INSPIRE]. 